\title{
Overview of piezoelectric energy harvesting technology in the tire condition monitoring systems
}

\author{
Rajae Alla ${ }^{1, *}$, Abdelmajid Bybi $^{2}$, Ayoub Benhiba $^{1}$, Hilal Drissi ${ }^{1}$ and El Ayachi Chater ${ }^{1}$ \\ ${ }^{1}$ Mohammed V University in Rabat, Higher School of Technology in Sale, LASTIMI, Salé, Morocco \\ ${ }^{2}$ Mohammed V University in Rabat, Higher School of Technology in Sale, MEAT, Salé, Morocco
}

\begin{abstract}
Over the past decade, energy harvesting from the surrounding resources has been a hot topic for numerous researches. The automotive industry is one field that pays attention to this clean energy which plays a primary role in the vehicle's safety. Moreover, a cost-efficient monitoring structure to better track tires conditions is required to fill the tires safety requirements. The main objective of this paper is to present a state of the art about the techniques of vibrational energy harvesting from car's tires for the embedded self-power sensors and tires condition monitoring systems (TCMS).
\end{abstract}

\section{Introduction}

The increasing costs of fuel and environmental pollution caused by fossil fuels require restricting the greenhouse effect and gasses emissions. Cars are recognized as one of the significant sources of greenhouse gasses emissions in the transportation sector. For this reason, researchers in the automotive industry have drawn attention to use clean and sustainable energy resources for vehicles sensors and equipment. Because the tires are the only interface between the vehicle and the road they play a central role in its safety. The automotive industry strives for a cost-efficient monitoring structure for autonomous vehicles to better track tires' conditions and ensure more safety for the passengers [1].

In standard, a tire condition monitoring system (TCMS) includes three essential parts which are the energy supply unit, a sensing unit, and a readout circuitry unit, as shown in Fig.1 [2].

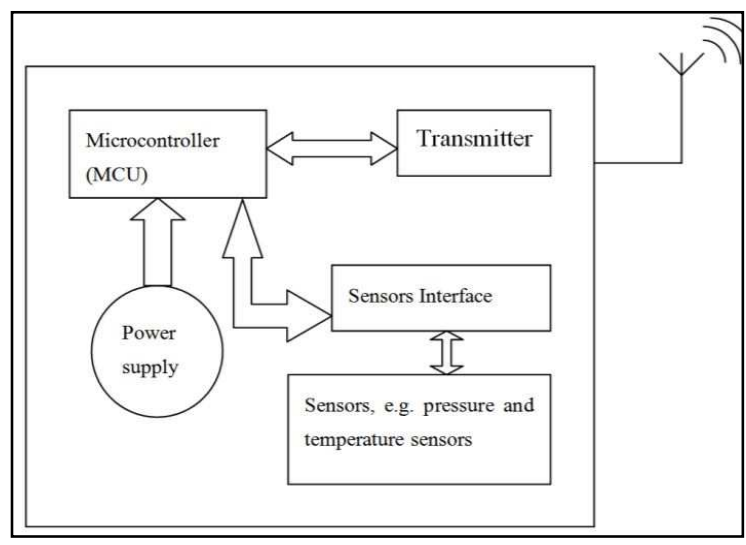

Fig. 1. TCMS schematic structure [2].

\footnotetext{
Rajae Alla: rajae.alla@gmail.com
}

The TCMS offers online measurement of many parameters referring to tires state, including their pressure, temperature, and road friction. These measurements increase automobile and passenger's safety [3]. Thus, a tire equipped with a TCMS is called intelligent tire. The implementation of smart tires is especially positive for reducing the number of accidents, $\mathrm{CO} 2$, noise emissions, and additionally fuel consumption [4]. To attempt the above-mentioned objectives, several researchers propose numerous sensory technologies to collect strain and deflection related data from tires [5].

As the TCMS needs a power supply to function, the batteries constitute the main source of energy in the vehicle. Nevertheless, due to their environmental harmful usage and their lifespan limitation, an autopower sensing system is needed. The latter can be realised by the fusion of sensory structures and energy harvesters (e.g., piezoelectric transducers). This combination results in emerging new devices which have the objective of self-power sensing [4].

Historically, pneumatic tires started as an upgrade from solid rubber tires in the United Kingdom in the late 1800s. They had a small cross-section and high pressure and were mainly used in bicycle applications. Larger tires were debuted in the early 1920 s and used in the automotive industry. In the early 1950s, with advancements in rim design, tubeless tires were introduced. In the late 1960s, Bias belt tires were promoted. Radial tires were first introduced in Europe. They became famous in the United States in the early 1970 s, and now dominate the passenger car tires market [2].

This paper is organized in five sections. The second one describes the car's tires and its different elements. The third section is dedicated to tires' sensing and energy harvesting technics. Section 4 deals about the evolution from a conventional tire sensing technology to 
a self-power sensing technology for TCMS thanks to energy harvesting $(\mathrm{EH})$ techniques particularly the inertia-based $\mathrm{EH}$ and strain EH. Conclusions are presented in section 5 .

\section{Tire description}

The main role of car tires is to provide the interface between the vehicle and the pavement. They support vehicle load and absorb road irregularities, i.e. tires perform the role of the spring and damper to absorb the irregularities of the pavement [6]. Cars' tires are mainly cord rubber composites which consist of rubber compound; reinforcement system and radial tire component composed of tread, bead, sidewall, shoulder, and ply [7]. The whole tire parts are described in fig. 2 .

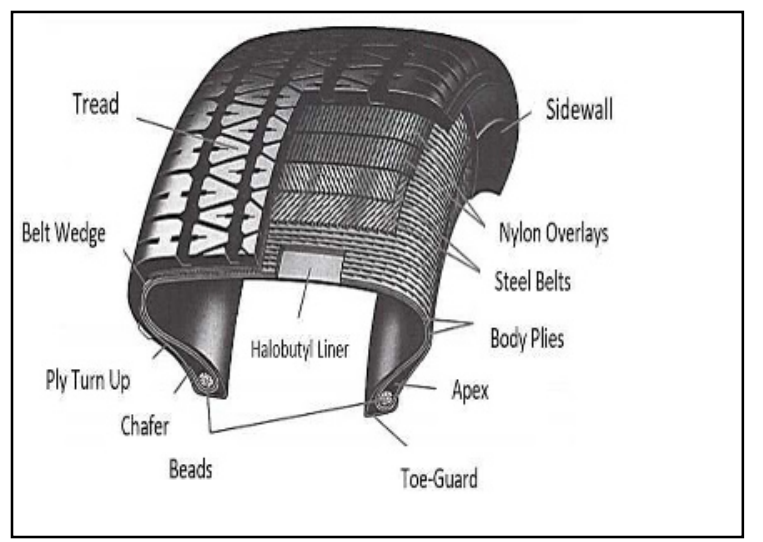

Fig. 2. Tire's elements [8].

\section{Tires sensing and energy harvesting technics}

Installing sensors inside the tires has become highly demanded to improve vehicle control and safety. Tire sensing grants direct measurement information and quantification about tire-pavement interactions, such as tire pressure monitoring system (TPMS), braking distance, contact path length, friction coefficient, slip angle, road conditions, and tire wear [1].

Recently, low-cost tire condition monitoring systems (TCMS) have become broadly hooked up into massproduced cars in both Europe and USA. The vehicles are equipped with a TPMS to alert drivers about the low strain and related higher fuel intake [9].

TPMS contains a TPM sensor installed into the back of the valve stem of each tire (fig.3), a TPM electronic control unit (ECU), a receiving unit Dashboard TPM warning light, and one or four antennas connected to the receiving unit. It transmits the pressure and temperature measurements and their identifiers [10]. Sustained TPMS is important to keep away from the battery-supply shortcomings. Thus, energy harvesting systems are substantially promising to reach this aim. Usually, the energy consumption of the common TPMS chips available in the market is in average of $0.45 \mathrm{~mW}$ to acquire a $1 \mathrm{~Hz}$ transmission rate [12].

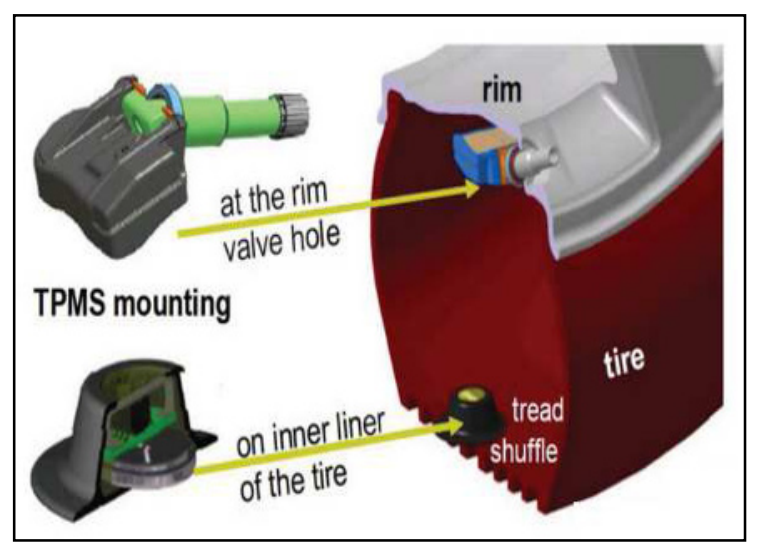

Fig. 3. TPMS installation inside the tire [11].

Passive and active sensors may be utilized in monitoring tires. Active sensors need an electrical power supply which is often a battery. The drawback of batteries is their main limited lifespan and may have a negative impact on the environment. Also, these sensors need maintenance to check and change their batteries which can be difficult when the sensor is placed in inaccessible zone. Similarly, the heat generated by the batteries' Joule effect may also affect the sensors' efficiency. All those problems increase the maintenance cost of intelligent tires [1]. For these reasons, it is interesting to find other solutions to make the active sensors autonomous, e.g. by recovering the surrounding energy such as vibrations energy in the case of TPMS sensors for tires.

\section{To a self-powered sensor for TCMS}

Using self-powered sensors for TCMS becomes the objective of numerous researches. The new challenge is to use the wasted kinetic energy sources for power scavenging. Several innovative proposals aim to extract clean beneficial energy from accessible ambient resources [4]. The most apparent energy sources in a shifting vehicle's tire inertia-based structure are associated with whirling and applying to cantilever structures. Moreover, the tire's strain energy is related to its cyclic deformation due to the contact with the pavement. The principal energy source used in the first type is the acceleration exchange of different points on a rolling tire, either on the wheel rim or on the tire liner, originated from the contact between the tires and the road. This acceleration amplitude tremendously depends on the importance of riding velocity. The power spectral density of the tire acceleration at a speed of $50 \mathrm{~km} / \mathrm{h}$ shows a rich spectrum. At low frequencies $(5-20 \mathrm{~Hz})$, there is a sturdy peak corresponding to the revolution length of the wheel [7].

Strain-based systems take advantage of the longitudinal strain that develops in a tire while it deforms due to the tire-read contact. The strain-type energy harvester requires more extensive elastic deformation because the energy generation relies on the applied strain. Strain-type energy harvesters have the 
benefits of ease of manufacturing, assembly, and structural integrity [8].

Various types of power harvesters for intelligent tire applications are proposed in the literature, and typically, they may be categorized into electromagnetic, electrostatic, and piezoelectric harvester systems. Compared with the piezoelectric ones, the electromagnetic and electrostatic structures have lower output voltage. Similarly, electrostatic harvesters demand an external voltage supply, and the embedding of electromagnetic energy harvesters with electronics and microsystems are complex.

\subsection{Inertia-based piezoelectric energy harvesters (PEH)}

The vibration-type energy harvesters using a piezoelectric cantilever beam and a tip mass as a mass-spring structure are one of the well-regarded configurations. This kind of energy harvester provides a power output within a range of 2.5 to $349 \mu \mathrm{W}$ [13].

As shown in Fig. 4, Gu and Livermore [14] suggested a self-tuning piezoelectric energy harvester for rotating applications.

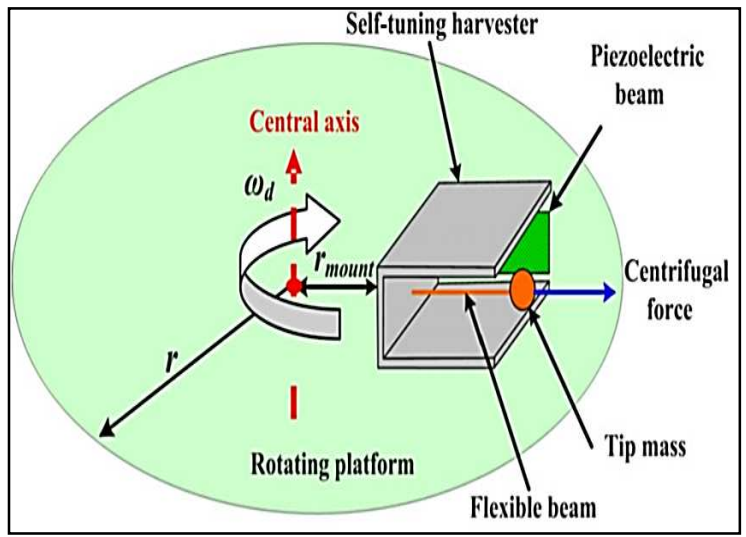

Fig. 4. Self-tuning PEH for rotating applications [14].

The harvester rotates in the vertical plane and is composed of two beams: a rather inflexible piezoelectric generating beam and a slim, flexible driving beam with a tip mass established at the quit. The system is able to generate a maximum power density about 30.83 $\mu \mathrm{W} . \mathrm{cm}^{-3}$. The piezoelectric energy harvester was designed for a tire with a $572 \mathrm{~mm}$ outer diameter. The velocity scale of the considered tires measures is from 25 miles per hour (mph) to $65 \mathrm{mph}$. This speed variety fits to a rotational frequency range from $6.2 \mathrm{~Hz}$ to $16.2 \mathrm{~Hz}$.

The main difficulty of this technique is the requirement of adjustment between the cantilever beam resonant frequency and the excitation frequency. Also, this sort of power harvesting method can simply harvest the vibration energy in one direction, whilst basically a tire undergoes a multi-directional deflection. Because of those challenges, the use of strain-primarily based strength harvesting systems is usually recommended [1].

\subsection{Strain-based PEH}

Ever since Apollo project [15] in 2005, several strain- based PEH were studied for tire applications.

Two processes had been considered. In the first one a PZT bender was immediately bonded to the internal surface of the tire. The second technique used tinier and rigid elements that produce charge due to a compressive load at the tire rim. Both PZT ceramics and Polymeric Polyvinylidene Fluoride (PVDF) materials were utilized inside tires. While the piezoelectric bender becomes connected to the inner wheel, deformation of the tire at the contact area leads to a cyclic deformation and consecutive relaxation because it leaves the contact patch [16]. PZT benders were chosen as they have been capable of enduring the large deflection of the tire. Bowen and Arafa reviewed the PEHs used for powering up the TPMS and reported that the power output of the strain based PEHs is in the range between $40 \mu \mathrm{W}$ and 6.5 $\mathrm{mW}$ [11]. Xie et al. [17] developed a new ring piezoelectric harvester stimulated by magnetic forces. The author indicated that the proposed ring could produce an electrical power up to $5274.8 \mathrm{~W}$. Xi and Wang [18] presented a theoretical model of a piezoelectric ring tire harvester via recurrence, which is manufactured from a sequence of discrete PZT4 patches in a square form circumferentially embedded in a polymer ring uniformly. As shown in Fig. 5, the harvester was positioned among the metal belts tightly stuck in the inside liner of tires.

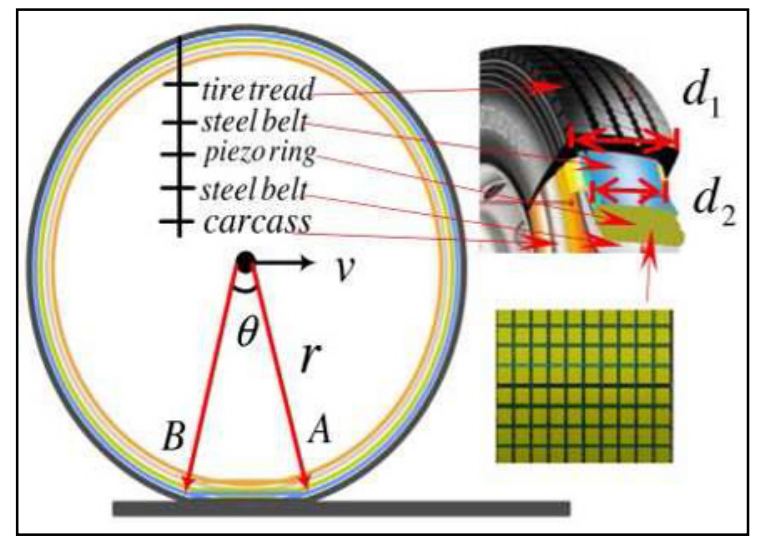

Fig. 5. Piezoelectric ring embedded in the tire [18].

The results showed that up to $42.08 \mathrm{~W}$ of electrical power can be harvested, for piezoelectric ring with the dimensions: radius, width, thickness, and range of the PZT4 pieces inside the direction of the tire width being $30,0.25 \mathrm{~m}, 0.01 \mathrm{~m}, 0.01 \mathrm{~m}$ and 3 , respectively. It is miles apparent that the output power of the strain-type PEHs is significantly better than that of the vibration-based technology used for tires. In the same context, Eshghi et al. [19] designed a TPMS energy harvester $(\mathrm{EH})$ consisting of a beam clamped at both ends with the PZT material attached on the top surface.

The EH was wrapped in a housing and attached to the tire's inside surface. The design focused on the radial acceleration which has the most important magnitudes. 
Lee et al. [20] suggested a model of energy harvester using the vertical deformation from the vehicle weight and inner liner deformation while driving. A piezoelectric composite containing fibers was utilized as a transducer converting mechanical energy into electric one. The electrical power acquired from a harvester with dimensions $60 * 10 * 0.3 \mathrm{~mm}^{3}$ for 1 cycle with $500 \mathrm{~kg}$ at a speed of $60 \mathrm{~km} / \mathrm{h}$ was about $1.37 \mu \mathrm{W} / \mathrm{mm}^{3}$. Esmaeeli et al. [1] reported a rainbow-shaped piezoelectric harvester capable of catching multidirectional deflections. This model comprises a flexible metal substrate layer and two piezoelectric layers, which are sandwiched and stuck together. The results of simulations proved that the maximum output voltage, electric power, and electric energy of the designed piezoelectric energy harvester are $9.7 \mathrm{~V}, 5.85 \mathrm{~mW}$, and $95 \mu \mathrm{J} / \mathrm{rev}$, respectively. Esmaeeli et al. [21] brought a brand-new design of strain-based energy harvester for the tire application prompted from a cymbal PEH. As shown in Fig. 6, the energy harvester includes two metallic hosts and a soft PZT-5H piezoelectric fabric layer.

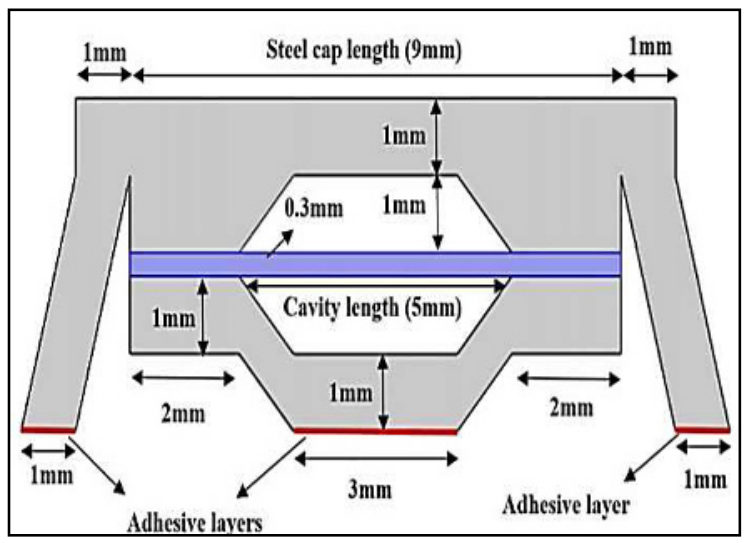

Fig. 6. Piezoelectric energy harvester inspired from a cymbal model [21].

The computed results indicated that the maximum output voltage and the maximum electric power are approximately $3.5 \mathrm{~V}$ and $2.8 \mathrm{~mW}$, respectively. These quantities might be sufficient to power two sensors. Maurya et al. [22] studied a PEH using a flexible organic piezoelectric material PVDF for continuous energy harvesting and surveying of the variable strain for a tire under special driving situations. An electrical power output of about $580 \mu \mathrm{W}$ is obtained at a frequency of 16 $\mathrm{Hz}$ and driving speed of $112 \mathrm{~km} / \mathrm{h}$. Yi et al. [12] proposed a rotation-driven piezoelectric energy harvester with eight traditional nonlinear buckled-bridges to extremely scavenge low-frequency kinetic power. Thinned bulk PZT film is employed as piezoelectric functional layer based on its electromechanical aspect. Gear induced oscillation mechanism guarantees the powerful deformation of the piezoelectric buckled bridges. The evolved harvester was able to generate an output power of $8.9 \mathrm{~mW}$ with an optimal resistance of 3 $\mathrm{k} \Omega$ at a rotational frequency of $8.3 \mathrm{~Hz}$.

In line with the Apollo project reviews about the intelligent tires [23], the sensors and systems are not fit to insert into tires because of the severe rolling actions. Also, the compatibility with rubber tires and durability problems are worth being investigated. Consequently, some different locations for the energy harvesters $(\mathrm{EH})$ were suggested in the literature, e.g. install them on the rim. In this context, Wu et al. [24] reported a seesawbased PVDF energy harvester installed on the rim tread and get an electrical power of $5.625 \mu \mathrm{W}$ at $750 \mathrm{rev} / \mathrm{min}$. Roundy and Tola [25] proposed an offset pendulum harvester mounted on the wheel to power a radio frequency transmission. The authors' road tests demonstrated that the proposed harvester was able to support better than one transmission per minute for speed variation from 10 to $155 \mathrm{~km} / \mathrm{h}$, and more than three transmissions per minute in the speed range of 32$155 \mathrm{~km} / \mathrm{h}$. Because the device is set up between the tire and the rim it is not handy for maintenance. Yu-Jen et al. [26] proposed a piezoelectric energy harvester with a trapezoidal cantilever installed at the outer surface of the rim, which is appropriate at speeds ranging from 200 to $700 \mathrm{rev} / \mathrm{min}$. The authors demonstrated that their prototype can harvester a power in the range of $70-140$ $\mu \mathrm{W}$ when connected to a 3.3 $\mathrm{M} \Omega$ resistor. Rui et al. [27] suggested a piezoelectric cantilever beam energy harvester set up within the rim spoke and boasts the capability of self-tuning due to the centrifugal force in the rotational motion (Fig. 7). An output electrical power in the range of $0.61-6.28 \mathrm{~mW}$ was harvested at a speed varying from 40 to $120 \mathrm{~km} / \mathrm{h}$ on a real road test.

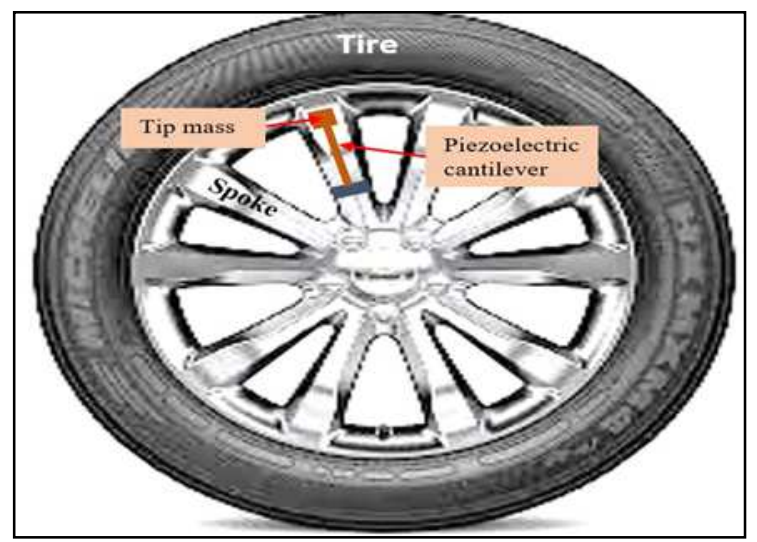

Fig. 7. Intelligent wheel spoke piezoelectric energy harvester (IWSPEH) [27].

The review of the literature about the piezoelectric energy harvesting and sensing devices in TCMS shows that there are some drawbacks and boundaries in their designs:

A) It is vital to design a harvester which could tune the frequency.

B) In the beam-mass model, the evolved devices ought to have overload protection.

C) The considered resonant frequency for the special advanced piezoelectric harvesting devices is not always realistic. 
D) The piezoelectric ceramics generally used in the energy harvesters are fragile.

Finally, even though the electric output of the actual piezoelectric sensors is sufficient for both power harvesting and sensing, they are still difficult to be prototyped in tires' systems because of their low thermal and mechanical sturdiness [4]. Consequently, solutions are needed to design and manufacture robust piezoelectric energy harvesters which both overcome the previous limitations and contribute to tires' sensors autonomy and passengers' safety.

\section{Conclusion}

This paper reviews the energy harvesting technology in sensing systems for vehicles. It focuses on the vibrational energy harvesting for the tires' condition monitoring systems (TCMS) and exhibit different types of piezoelectric energy harvesters (PEH). The main objective is to propose solutions to make the systems self-power by harvesting the surrounded vibrational energy usually lost in the tires. From the state of the art, it is found that the majority of harvesting piezoelectric devices are still presenting limitations, i.e. mainly their difficulty of integration in real moving tire and the limited harvested electrical energy. The challenge is layout strong energy harvesting structures to face up to the harsh conditions for real case and combine the component of the harvester in one platform to installed it in a suitable emplacement. As a perspective of this research work, theoretical and simulation studies will be conducted to investigate energy harvesting by placing piezoelectric elements outside the tire, e.g. install them on the rim.

\section{References}

1. R. Esmaeeli, H. Aliniagerdroudbari, S. R. Hashemi, A. Nazari, M. Alhadri, W. Zakri, et al., "A rainbow piezoelectric energy harvesting system for intelligent tire monitoring applications," Journal of Energy Resources Technology, vol. 141, (2019).

2. E. Kubba and K. Jiang, "A comprehensive study on technologies of tyre monitoring systems and possible energy solutions," Sensors, vol. 14, pp. 10306-10345, (2014).

3. H. Askari, Z. Saadatnia, A. Khajepour, M. B. Khamesee, and J. Zu, "A triboelectric self-powered sensor for tire condition monitoring: concept, design, fabrication, and experiments," Advanced Engineering Materials, vol. 19, p. 1700318, (2017).

4. H. Askari, E. Hashemi, A. Khajepour, M. B. Khamesee, and Z. L. Wang, "Tire condition monitoring and intelligent tires using nanogenerators based on piezoelectric, electromagnetic, and triboelectric effects," Advanced Materials Technologies, vol. 4, p. 1800105, (2019).
5. R. Matsuzaki and A. Todoroki, "Wireless flexible capacitive sensor based on ultra-flexible epoxy resin for strain measurement of automobile tires," Sensors and Actuators A: Physical, vol. 140, pp. 32-42, (2007).

6. A. N. Gent and J. D. Walter, "Pneumatic tire" Mechanical Engineering Faculty Research. 854, (2006).

7. B. Rodgers and W. Waddell, "The science of rubber compounding," in science and technology of rubber, ed: Elsevier, pp. 401-454, (2005).

8. B. Rodgers and W. Waddell, "Tire engineering," in Science and Technology of Rubber, ed: Elsevier, pp. 619-II, (2005).

9. O. J. Jousimaa, Y. Xiong, A. J. Niskanen, and A. J. Tuononen, "Energy harvesting system for intelligent tyre sensors," IEEE Intelligent Vehicles Symposium (IV), 2016, pp. 578-583. (2016)

10. I. Rouf, R. D. Miller, H. A. Mustafa, T. Taylor, S. $\mathrm{Oh}, \mathrm{W} . \mathrm{Xu}$, et al., "Security and Privacy Vulnerabilities of In-Car Wireless Networks: A Tire Pressure Monitoring System Case Study," in USENIX Security Symposium, (2010).

11. C. Bowen and M. Arafa, "Energy harvesting technologies for tire pressure monitoring systems," Advanced Energy Materials, vol. 5, p. 1401787 , (2015).

12. Z. Yi, B. Yang, W. Zhang, Y. Wu, and J. Liu, "Batteryless tire pressure real-time monitoring system driven by an ultralow frequency piezoelectric rotational energy harvester," IEEE Transactions on Industrial Electronics, vol. 68, pp. 3192-3201, (2020).

13. A. E. Kubba and K. Jiang, "Efficiency enhancement of a cantilever-based vibration energy harvester," Sensors, vol. 14, pp. 188-211, (2014).

14. L. Gu and C. Livermore, "Compact passively selftuning energy harvesting for rotating applications," Smart materials and structures, vol. 21, p. 015002, (2011).

15. Y.-J. Wang, C.-D. Chen, and C.-K. Sung, "Design of a frequency-adjusting device for harvesting energy from a rotating wheel," Sensors and Actuators A: Physical, vol. 159, pp. 196-203, (2010).

16. N. Makki and R. Pop-Iliev, "Battery-and wire-less tire pressure measurement systems (TPMS) sensor," Microsystem technologies, vol. 18, pp. 1201-1212, (2012).

17. X. Xie, Q. Wang, and N. Wu, "A ring piezoelectric energy harvester excited by magnetic forces," International Journal of Engineering Science, vol. 77, pp. 71-78, (2014).

18. X. Xie and Q. Wang, "A mathematical model for piezoelectric ring energy harvesting technology from vehicle tires," International Journal of Engineering Science, vol. 94, pp. 113-127, (2015). 
19. A. T. Eshghi, S. Lee, M. K. Sadoughi, C. Hu, Y.-C. Kim, and J.-H. Seo, "Design optimization under uncertainty and speed variability for a piezoelectric energy harvester powering a tire pressure monitoring sensor," Smart Materials and Structures, vol. 26, p. 105037, (2017).

20. J. Lee and B. Choi, "Development of a piezoelectric energy harvesting system for implementing wireless sensors on the tires," Energy conversion and management, vol. 78, pp. 32-38, (2014).

21. R. Esmaeeli, H. Aliniagerdroudbari, S. R. Hashemi, M. Alhadri, W. Zakri, C. Batur, et al., "Design, modelling, and analysis of a high-performance piezoelectric energy harvester for intelligent tires," International Journal of Energy Research, vol. 43, pp. 5199-5212, (2019).

22. D. Maurya, P. Kumar, S. Khaleghian, R. Sriramdas, M. G. Kang, R. A. Kishore, et al., "Energy harvesting and strain sensing in smart tire for next generation autonomous vehicles," Applied energy, vol. 232, pp. 312-322, (2018).

23. T. Makinen and H. Wunderlich, "Intelligent tyre promoting accident-free traffic," in Proceedings. The IEEE 5th International Conference on Intelligent Transportation Systems, pp. 606-609, (2002).

24. X. Wu, M. Parmar, and D.-W. Lee, "A seesawstructured energy harvester with super wide bandwidth for TPMS application," IEEE/ASME Transactions on Mechatronics, vol. 19, pp. 15141522, (2013).

25. S. Roundy and J. Tola, "Energy harvester for rotating environments using offset pendulum and nonlinear dynamics," Smart Materials and Structures, vol. 23, p. 105004, (2014).

26. W. Yu-Jen, C. Tsung-Yi, and Y. Jui-Hsin, "Design and kinetic analysis of piezoelectric energy harvesters with self-adjusting resonant frequency," Smart Materials and Structures, vol. 26, p. 095037 , (2017).

27. X. Rui, Z. Zeng, Y. Zhang, Y. Li, H. Feng, X. Huang, et al., "Design and experimental investigation of a self-tuning piezoelectric energy harvesting system for intelligent vehicle wheels," IEEE Transactions on Vehicular Technology, vol. 69, pp. 1440-1451, (2019). 